This item was submitted to Loughborough's Research Repository by the author.

Items in Figshare are protected by copyright, with all rights reserved, unless otherwise indicated.

\title{
End fixity to spiral strands undergoing cyclic bending
}

PLEASE CITE THE PUBLISHED VERSION

PUBLISHER

Professional Engineering Publishing / @ IMechE

VERSION

VoR (Version of Record)

LICENCE

CC BY-NC-ND 4.0

REPOSITORY RECORD

Raoof, Mohammed, and T.J. Davies. 2019. "End Fixity to Spiral Strands Undergoing Cyclic Bending”. figshare. https://hdl.handle.net/2134/4755. 
This item was submitted to Loughborough's Institutional Repository (https://dspace.lboro.ac.uk/) by the author and is made available under the following Creative Commons Licence conditions.

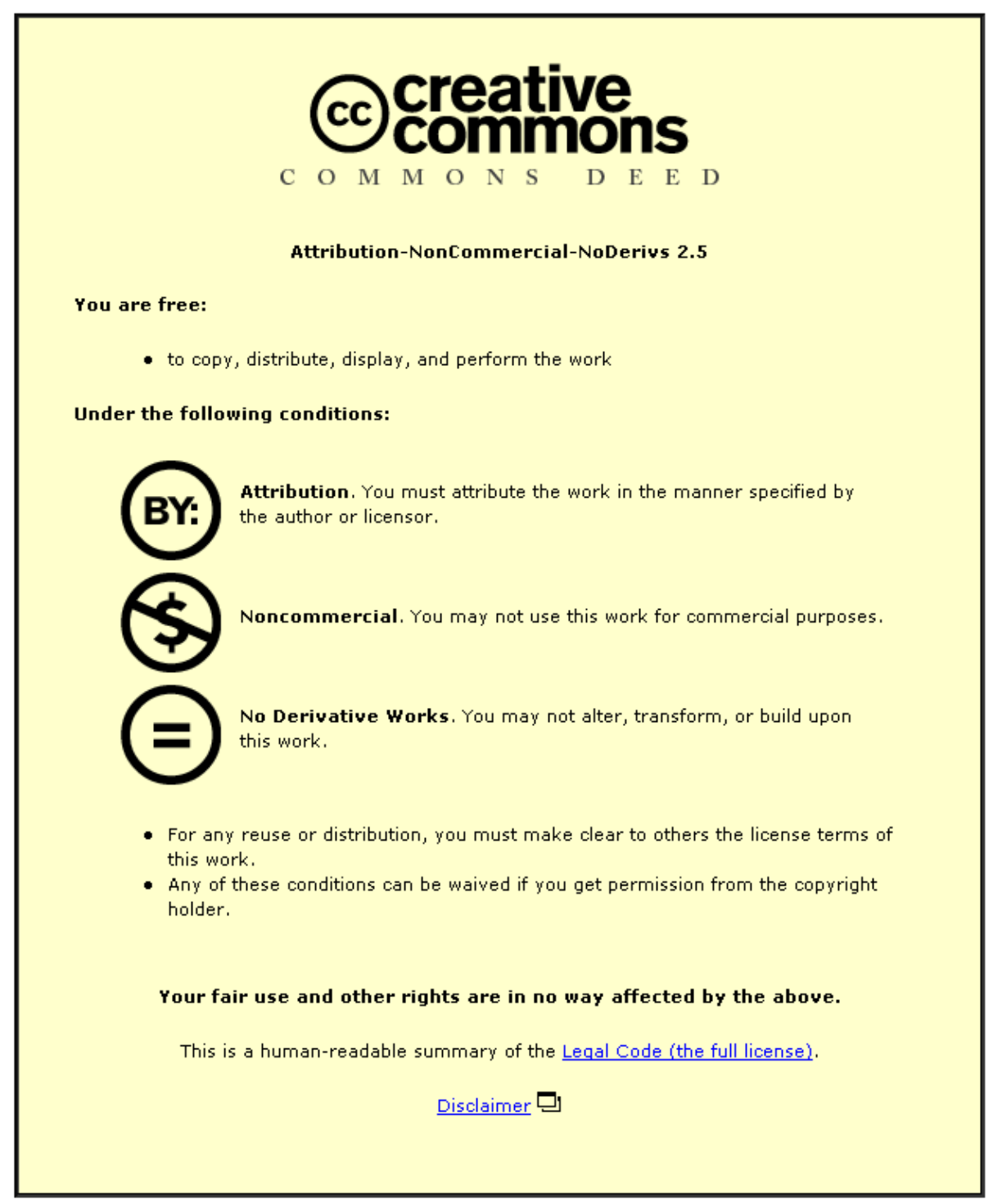

For the full text of this licence, please go to: http://creativecommons.org/licenses/by-nc-nd/2.5/ 


\title{
End fixity to spiral strands undergoing cyclic bending
}

\section{Raoof* and T J Davies}

Civil and Building Engineering Department, Loughborough University, Loughborough, Leicestershire, UK

The MS was received on 7 January 2004 and was accepted after revision for publication on 13 September 2004.

\author{
DOI: $10.1243 / 030932405 X 7746$
}

\begin{abstract}
Based on a semi-empirical approach and guided by the previously reported experimental observations and theoretical work by the first author, it is suggested that for spiral strands with mean axial strains $\varepsilon_{\mathrm{c}}>0.0025$ and outside diameters $d \leqslant 40 \mathrm{~mm}$, planesection bending may reasonably be assumed in the course of theoretically determining the deflected shape of the strands in the immediate vicinity of fixed end(s) with the proviso that, at the assumed ideal fixed end(s) to the spiral strands, $\rho / d>630$, where $\rho$ is the estimated minimum radius of curvature at the fixed end. It is also shown that, in practice, the effective fixed end to the spiral strands undergoing such cyclic bending is located not at the face of the zinc-socketed termination, but well within the socket itself; for an axially preloaded spiral strand of $39 \mathrm{~mm}$ outside diameter, the effective point of end fixity is shown to be located inside the conical housing, about $60 \mathrm{~mm}$ (or, say, 1.5 diameters) away from the face of the socket. The practical implications of the present findings as regards the traditional approach(es) for determining the minimum radii of curvature at the theoretically assumed (ideal) fixed end, the exact location of which in practice has invariably not (in the previous literature) been properly defined, are critically discussed with such estimated magnitudes of minimum radii of curvature often used as an input into various available models for design against spiral strand restrained bending fatigue. It is, perhaps, worth mentioning that, in the present terminology, the term 'spiral strand restrained bending fatigue' refers to those cases whereby strand fatigue failures near partially (or fully) restrained terminations of various types occur as a result of cyclic bending of the strand at the termination due to, for example, hydrodynamic or aerodynamic loading.
\end{abstract}

Keywords: spiral strands, bending, cyclic loading, fatigue, bridges, offshore platforms, guyed masts, overhead conductors

\section{INTRODUCTION}

Over the past 20 years, very substantial improvements have been made in the understanding of the behaviour of spiral strands. Spiral strand, or strand, is (in UK parlance) a group of wires laid helically in successive layers over a straight central king wire, with the individual wires in each layer $i$ in a spiral strand following a simple helical path with lay angle $\alpha_{i}$ [Fig. 1(a)].

This paper is concerned with certain aspects of bending effects in multilayered spiral strands in the absence of sheaves or other formers, so that the

*Corresponding author: Civil and Building Engineering

Department, Loughborough University, Loughborough,

Leicestershire LE11 3TU, UK; email: m.raoof@lboro.ac.uk radius of curvature of the strand is not predetermined. These conditions will be referred to as 'free bending'. Free-bending problems are a source of concern (and not infrequent failures) in structures ranging from floating offshore platforms, suspension and cable-stayed bridges, and the stays for guyed masts, to electromechanical cables where spiral strand fatigue failures near partially (or fully) restrained terminations of various types caused by, for example, hydrodynamic or aerodynamic loading are not uncommon.

For the free bending of long spiral strands under an approximately steady mean axial load, it is common to introduce a mathematically convenient constant effective bending stiffness $(E I)_{\text {eff }}$ for the strand, using which the radii of curvature at the points of restraint are calculated. Traditionally, the maximum 


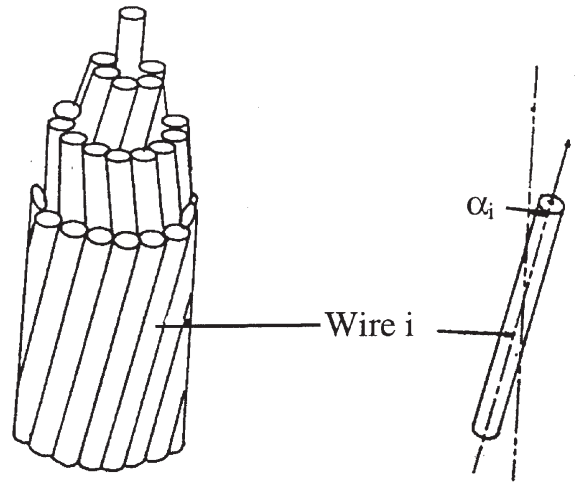

(a)

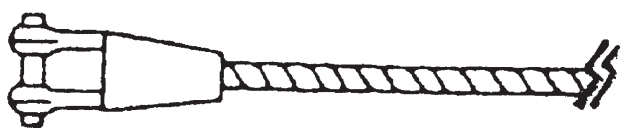

(b)

Fig. 1 (a) Multilayered spiral strand; (b) zinc-poured conical socket and spiral strand assembly used in the present experiments

bending strains have then been found on the basis of a variety of, frankly, sweeping assumptions; these strains have further been assumed (without any sound experimental and/or theoretical justification) to govern the strand bending fatigue life [1].

The validity of such maximum (extreme fibre) bending stress approaches (in terms of the simple beam bending theory), however, has fairly recently been questioned [1-4], and it has been demonstrated experimentally that the neutral axis (as opposed to the extreme fibre position) is the location where initial wire fractures under restrained cyclic bending invariably occur. Spiral strand restrained bending fatigue failures at terminations have been shown (by both theory and experiments) to be controlled by interwire/interlayer fretting which is greatest not at the extreme fibre position but in the vicinity of the so-called neutral axis where wire bending stresses are, indeed, minimal. A simple new design method against restrained bending fatigue has thus been developed [1]. There are, however, a number of still unresolved issues which need clarification and some aspects of these form the subject of the present paper.

As regards the spiral strand effective bending stiffness, due to their peculiar construction, axially preloaded spiral strands undergo plane-section bending only for sufficiently large radii of strand curvature. For radii of curvature below a certain limit, plane sections do not remain plane and interlayer slippage takes place, starting from the outer layer, and spreading inwards, towards the centre of the strand, depending on the level of axial tension and imposed radius of curvature $[5,6]$. Indeed, in view of the small radii of curvature at points of fixity to spiral strands, it is debatable whether the traditional method of assuming a constant effective bending stiffness for theoretically predicting the minimum radii of curvature at fixed terminations is a reasonable approach, providing accurate predictions to be used as an input into the subsequent design calculations against restrained bending fatigue.

A series of carefully conducted and large-scale experiments on an axially preloaded spiral strand of $39 \mathrm{~mm}$ outside diameter have already been reported by Raoof [5] who used the Poffenberger-Swart differential displacement method [7] (a well-known technique in the field of overhead transmission lines) to measure the spiral strand deflected shapes in the immediate vicinity of nominally fixed zinc-poured sockets.

In the present paper, by using the test data of reference [5] in conjunction with an extended version of the simple formulations originally proposed by Wyatt [8], it will be demonstrated that, under certain conditions to be reasonably identified in what follows, the traditional assumption of a constant plane-section effective bending stiffness is, in fact, reasonable for determining the minimum radii of curvature at points of fixity to axially preloaded spiral strands undergoing cyclic bending with maximum lateral deflection-span ratios similar to those occurring under, say, vortex shedding instabilities. Moreover, it will be shown that, in practice, the effective fixed end is located not at the face of the zinc-socketed termination but well within the socket itself. These findings are believed to have significant practical implications on wire fretting and, therefore, fatigue positions in relation to spiral strand restrained bending fatigue design.

\section{EXPERIMENTAL OBSERVATIONS}

Full details of the carefully conducted large-scale experiments have been reported elsewhere [5], and only a few of the most relevant observations will be repeated here for completeness: these will then enable the reader to understand (and appreciate) better the subsequent theoretical developments.

The free-bending tests were carried out on an axially preloaded spiral strand $7.9 \mathrm{~m}$ long and of $39 \mathrm{~mm}$ outside diameter with the spiral strand construction details given in reference [9]. In these experiments, the specimen was subjected to a state of loading, as shown schematically in Fig. 2.

The strand was terminated at both ends with zincpoured sockets to BS 463 [10], except for an elongated jaw. Very briefly, in this socketing procedure, the spiral strand is seized, to prevent unwinding, cut, 
Position of lateral jacks

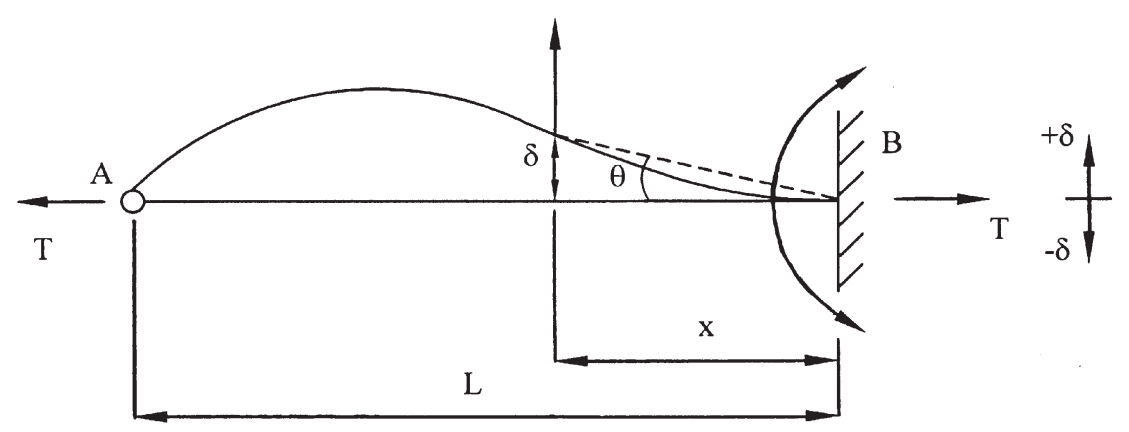

Fig. 2 Deflected shape of an axially preloaded spiral strand subjected to a single lateral point load

passed through the neck of the socket, brushed and thoroughly cleaned; once the socket is in position and the brush drawn in, the molten zinc is poured into the socket and allowed to solidify, with the final socket-strand assembly shown in Fig. 1(b).

In the course of the experiments, the socket at the axially fixed hold-down (position A in Fig. 2) was mounted with its pin vertical. The lateral exciter was placed at $x=2530 \mathrm{~mm}$ from the face of the other socket (whose pin was horizontal), at the position of the moving cross-head (i.e. position B). Four substantial box sections were welded to the base plate here so that the slightly flexible socket with a horizontal pin could be propped against them and, hence, a nominally fixed end against lateral movements could be achieved. It should be noted that as regards the position $\mathrm{A}$, due to the possible movement on the pin and the socket/strand flexibility at this position, as against the large distance from the lateral applied displacement, such relative rotational freedom at position A has negligible effects on the overall deflected shape in the region of interest at position $B$, with the specific value of $x=2530 \mathrm{~mm}$ determined by the fixed location in the laboratory of the prestressing floor bolts used for connecting the lateral jacks to the strong floor. Following Poffenberger and Swart [7], a rigid arm was extended from the socket, at position B, along which a series of closely spaced displacement transducers of $\pm 5 \mathrm{~mm}$ range were positioned. This arrangement was designed to provide reliable estimates of net strand lateral deflections (differential displacements) in the vicinity of the nominally fixed socket, free from any rigid body effects [5].

In the present tests, the free bending modes are classified as $(-\delta),(+\delta)$, and $( \pm \delta)$, where the modes $(-\delta)$ and $(+\delta)$ correspond to cases where the strand is cycled to one side, while type $( \pm \delta)$ identifies a reversed bending process. The positive sign represents the case in which the lateral strand deflection at the fixed socket is in the same direction as the lay angle in the outermost layer of wires, when looking along the strand from the cross-head (position B in Fig. 2) and from above.

Figure 3 shows the experimentally obtained deflected shapes for the $39 \mathrm{~mm}$ strand at the propped socket under modes $(-\delta)$ and $(+\delta)$ of free bending. The mean axial load was $0.41 \mathrm{MN}$ (corresponding to a mean axial strain $\varepsilon_{\mathrm{c}}=0.00287$ which is representative of the typical working conditions) with the spiral strand having a nominal ultimate tensile strength of 1.23 MN (i.e. three times the mean axial load). The lack of symmetry in these test results is not due to significant changes in the strand's effective bending rigidity (as, for example, confirmed by the individual axial wire strain measurements fully discussed in references [5] and [11]). Instead, it is believed to be partly due to the presence of external rigid body movements, as is demonstrated in Fig. 4. Here, the total deflection range of each transducer under modes $(-\delta)$ and $(+\delta)$ (i.e. $2 \delta$ ) is plotted against the total movement of the clamp at the lateral jack position (i.e. at $x=2530 \mathrm{~mm}$ in Fig. 2). In Fig. 4 , the corresponding points for the $( \pm \delta)$ mode are also superimposed on these. All these plots can be linearly extrapolated to very nearly a single point on the horizontal axis with an intercept of about $1 \mathrm{~mm}$. In other words, there appears to be an initial offset (probably due to the eccentricity as the strand enters the cone depending on the skill of the craftsman making the socket and other clearance problems), between the lateral strand deflection and the rigid body movement of the clamp at the lateral jack position. This, however, is not a serious pitfall and, as shown in the following, it may safely be bypassed for the present purposes. The plotted test data points in Fig. 3 clearly demonstrate that, irrespective of this secondary clearance problem, the strand lateral deflection at the face of the socket (i.e. at the strand-zinc interface) is not zero and, in fact, the point of zero lateral net deflection is somewhere well inside the conical housing. It is noteworthy that in Fig. 4, as the tension is constant, the linearity here suggests at once that the effective 


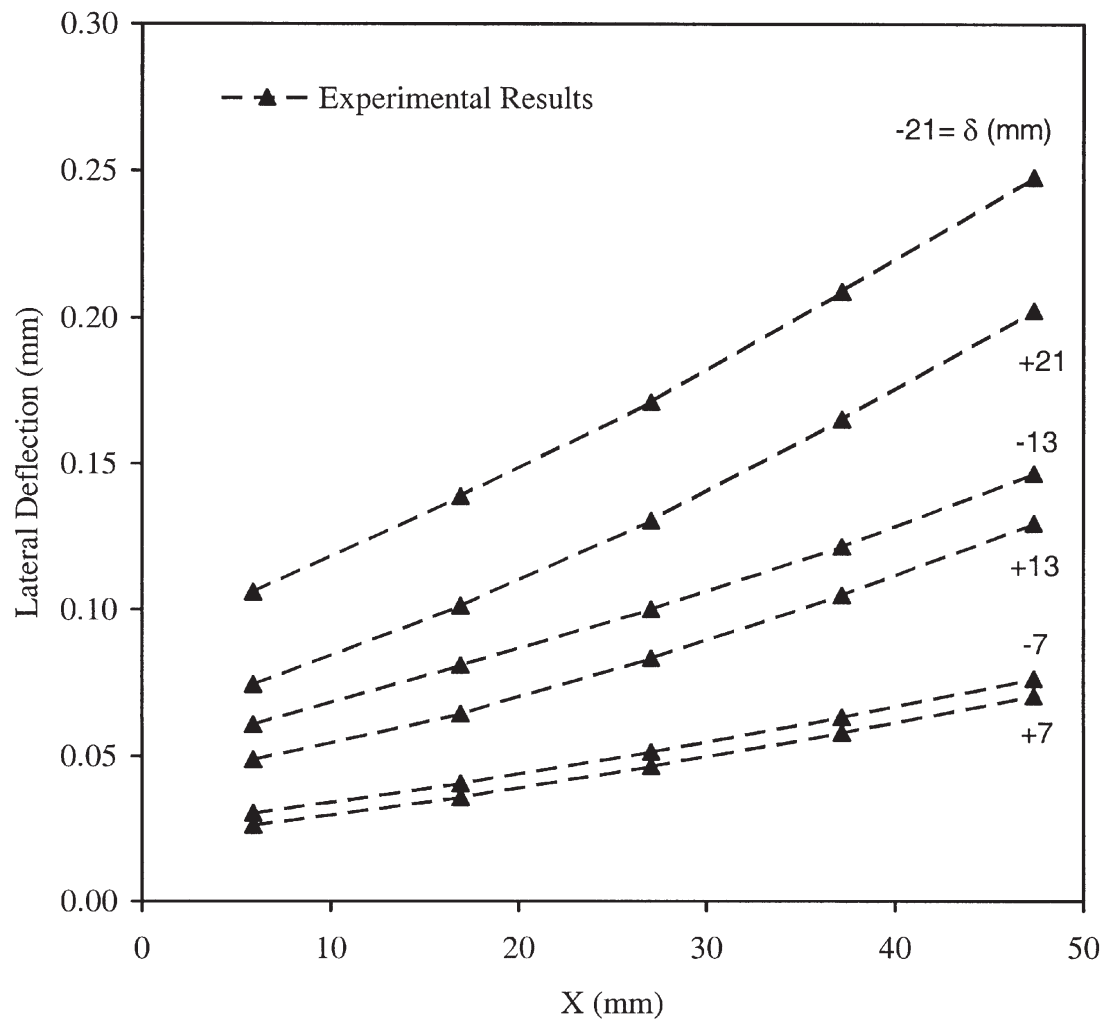

Fig. 3 The experimentally obtained deflected shapes for the $39 \mathrm{~mm}$ strand at the propped socket under modes $(-\delta)$ and $(+\delta)$ of free bending. (After reference [5])

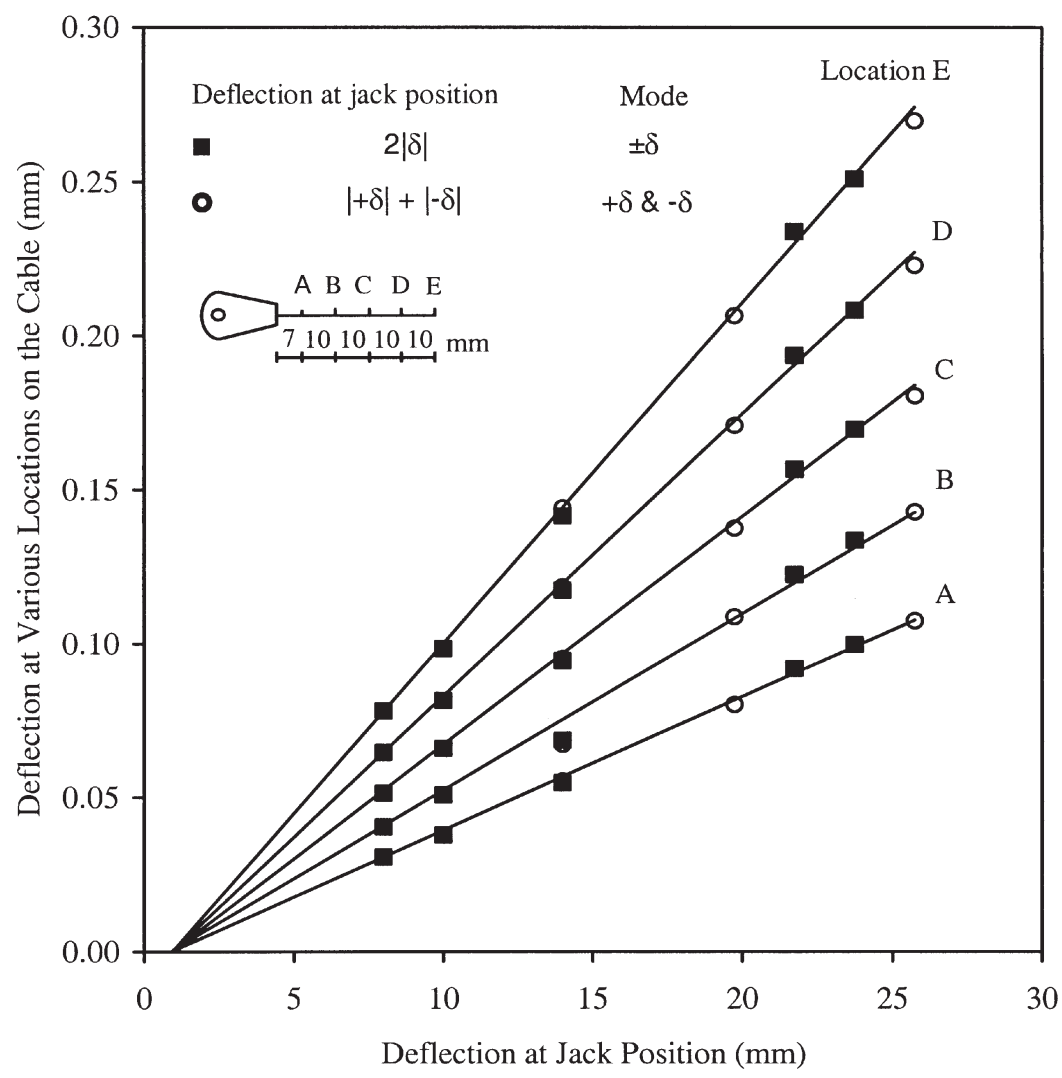

Fig. 4 Net deflection at various locations along the spiral strand of $39 \mathrm{~mm}$ outside diameter near the fixed socket obtained by the differential displacement method. (After reference [5]) 


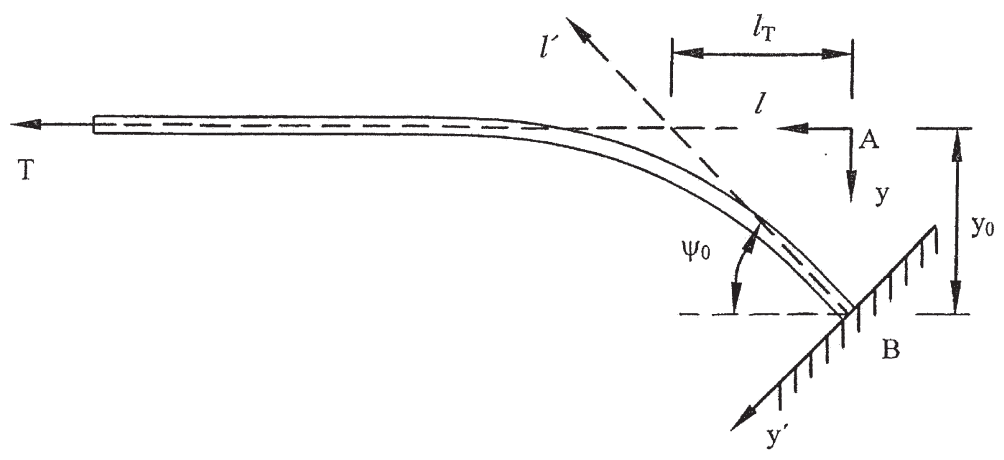

Fig. 5 Bending deflection of a simple tendon: the small deformations have been exaggerated for clarity of presentation

$E I$ is (for all practical purposes) the same for all the values of the displacement $\delta$ used in the tests; this is an important point as regards the following developments.

For the present purposes, the following section presents a simple semi-empirical method, based on the experimental data in Figs 3 and 4, for obtaining a reasonable estimate of the location, well within the zinc-poured socket, where the position of effective zero lateral strand deflection lies. This semiempirical method (which assumes small-deflection behaviour) will also enable critical examination of the validity of assuming a constant $(E I)_{\mathrm{eff}}$ in the course of theoretically determining the deflected shape of the axially preloaded spiral strand with its associated very rapid changes in radius of curvature in the immediate vicinity of the nominally fixed termination.

\section{THEORY}

\subsection{Local phenomena near the fixed end}

Back in 1960, Wyatt [8] considered the fundamental behaviour of a tendon carrying a large axial load and subjected to bending at one end as shown in Fig. 5. In this figure, the angle $\psi_{0}$ is the rotation of the tangent (say, at a termination) relative to the direction of the relevant tension $T$. Assuming smalldeflection behaviour, the moment equilibrium equation at any section then gives

$$
M=(E I)_{\mathrm{eff}} \frac{\mathrm{d}^{2} y}{\mathrm{~d} l^{2}}=T y
$$

where $(E I)_{\text {eff }}$ is the effective bending stiffness of the spiral strand, $M$ is the bending moment, and $l$ denotes the dimension measured along the strand. The solution to differential equation (1) is of the general form

$$
y=A \mathrm{e}^{g l}+B \mathrm{e}^{-g l}
$$

where

$$
g=\sqrt{\frac{T}{(E I)_{\mathrm{eff}}}}
$$

Based on the end conditions in Fig. 5, therefore, the constants $A(=0)$ and $B$, can be determined. Thus

$$
y=\frac{\psi_{0}}{g} \mathrm{e}^{-g l}
$$

Using equations (1) and (4), the radius of curvature at the fixed end, $\rho$, where $l=0$, is given as

$$
\frac{1}{\rho}=\psi_{0} \sqrt{\frac{T}{(E I)_{\mathrm{eff}}}}
$$

It should be noted that, in practice (under, say, vortex shedding instabilities) the angle $\psi_{0}$ is usually very small so that $\tan \psi_{0} \approx \psi_{0}$. Equation (4) suggests the presence of a characteristic length of the 'boundary layer' near the fixed end, of the order of one diameter, but clearly a function of the tension $T$ as well as $(E I)_{\text {eff }}[12]$.

For Raoof's experimental arrangement [5, 11], with the spiral strand subjected to a single transverse point load (Fig. 2), it has been shown [13] that the radius of curvature, $\rho$, at the fixed end may reasonably be calculated from

$$
\frac{1}{\rho} \approx 1.1 \frac{\delta}{x} \sqrt{\frac{T}{(E I)_{\mathrm{eff}}}}
$$

where $\delta / x=\tan \theta$, with the other parameters defined in Fig. 2. In particular, the difference between $\theta$ (which is shown in Fig. 2) and $\psi_{0}$ should be noted, with the latter being the rotation of the tangent at the termination relevant to the direction of the tension in the spiral strand.

Turning now to the new developments, in the experiments of reference [5], for a lateral deflection $\delta$ (at the lateral jack position) at a distance 
$x=2530 \mathrm{~mm}$ from the fixed end

$$
\tan \theta=\frac{\delta-1}{2530}
$$

where $\delta$ is in millimetres and, in equation (7), its magnitude is reduced by $1 \mathrm{~mm}$ (hence, the factor 1 in the numerator), because of the $1 \mathrm{~mm}$ intercept on the horizontal axis in Fig. 4, in order to take out any undesired effects of eccentricity, rigid-body movements, etc., in the following semi-empirical approach for determining the location of the effective point of fixity inside the socket.

Using equations (5) to (7), therefore, the equivalent angle of rotation, $\psi_{0}$, in Raoof's experiments, is

$$
\psi_{0} \approx \tan ^{-1}\left(1.1 \frac{\delta-1}{2530}\right)
$$

where, as mentioned previously, $\delta$ is in millimetres and $\psi_{0}$ is in radians. For reasons that become clear later, the $y$ and $l$ coordinate axes in Fig. 5 may be rotated through an angle $\psi_{0}$ to the alternative coordinate axes $y^{\prime}$ and $l^{\prime}$, through the relations

$$
\begin{aligned}
& y^{\prime}=l \sin \psi_{0}+y \cos \psi_{0} \\
& l^{\prime}=l \cos \psi_{0}-y \sin \psi_{0}
\end{aligned}
$$

Based on equations (4) and (9), then

$$
\begin{aligned}
& y^{\prime}=l \sin \psi_{0}+\left(\frac{\psi_{0}}{g} \mathrm{e}^{-g l} \cos \psi_{0}\right) \\
& l^{\prime}=l \cos \psi_{0}-\left(\frac{\psi_{0}}{g} \mathrm{e}^{-g l} \sin \psi_{0}\right)
\end{aligned}
$$

Finally, for $l=0$, equation (10a) gives

$$
y_{0}=\frac{\psi_{0}}{g} \cos \psi_{0}
$$

so that, with the origin of the coordinate system moved from point A in Fig. 5 to point B in this same figure

$$
y^{\prime}=\left[l \sin \psi_{0}+\left(\frac{\psi_{0}}{g} \mathrm{e}^{-g l} \cos \psi_{0}\right)\right]-y_{0}
$$

with $l^{\prime}$ again given by equation (10b) and, due to the very small magnitudes of $\psi_{0}$ in equation (10b), in practice $l \approx l^{\prime}$. Equations (12) and (10b) with $\psi_{0}$ given by equation (8), and $g$ defined by equation (3), then, define the deflected shape of the spiral strand in the immediate vicinity of the fixed end, with $y^{\prime}$ and $l^{\prime}$ representing the lateral deflection and longitudinal axis respectively of the spiral strand in the experimental set-up of references [5] and [11] (Fig. 2 ). However, it should be noted that, in equations (12) and (10b), the location of the precise point of fixity (i.e. as to whether it is at the face of the socket or well inside it) is not yet exactly defined, and this issue will be clarified later, using the experimental results presented in Fig. 3.

\section{RESULTS AND DISCUSSION}

The theoretical no-slip and full-slip values of planesection bending stiffness for the strand of $39 \mathrm{~mm}$ outside diameter are $1.513 \times 10^{10}$ and $1.279 \times 10^{10} \mathrm{~N} \mathrm{~mm}^{2}$ respectively $[\mathbf{9}]$. It should be noted at once that, as fully discussed by Raoof and Hobbs [2], even for a strand undergoing planesection bending, line-contact slippage between the adjacent wires in any given layer can take place, and hence the values of the effective bending stiffness for each layer do not stay constant; in the present terminology, the no-slip and full-slip values relate to cases when, in the presence of interwire friction, either the adjacent wires throughout the axially preloaded spiral strand stick together or gross sliding takes place over their lines of contact respectively, while the spiral strand undergoes plane-section bending. For a mean axial tension $T=0.41 \mathrm{MN}$, then the theoretical deflected shapes of the $39 \mathrm{~mm}$ strand in the vicinity of the nominally fixed zinc socket may be obtained, for different values of $\delta=7,13$, and $21 \mathrm{~mm}$, using the method developed in the previous section and by initially assuming the ideal point of fixity to be located at the face of the socket where $l^{\prime}=0$. Comparisons between the soobtained theoretical deflection curves and the experimental data, however, have suggested that, in order to obtain good correlations between theory and experiments, the theoretical plots should be shifted in a negative $l^{\prime}$ direction until encouraging agreement is found between theory and test data for all values of $\delta$. Figure 6 presents the outcome of such an exercise; by using the full-slip value of plane-section $(E I)_{\text {eff }}$ and by moving the theoretical plots in the negative $l^{\prime}$ direction by $58 \mathrm{~mm}$ (where $l^{\prime}=0$ denotes the strand-socket face), a very good match between theory and the average values of test data (i.e. the average of the test data corresponding to positive and negative values of $\delta$ in Fig. 3) over a wide range of $7 \mathrm{~mm} \leqslant \delta \leqslant 21 \mathrm{~mm}$ has been obtained. It is noteworthy that, assuming full-slip $(E I)_{\mathrm{eff}}$, for the most severe case in the present tests, i.e. when $\delta=21 \mathrm{~mm}$, equation (6) gives $\rho=19.3 \mathrm{~m}$.

It is, perhaps, worth mentioning that in the case when the no-slip value of $(E I)_{\text {eff }}$ was used for producing the theoretical curves, the same remarkably close correlations as in Fig. 6 were found between the theoretical predictions and the average values of the test data, with the point of zero lateral deflection found to be located at $l^{\prime}=-61 \mathrm{~mm}$ (inside the 


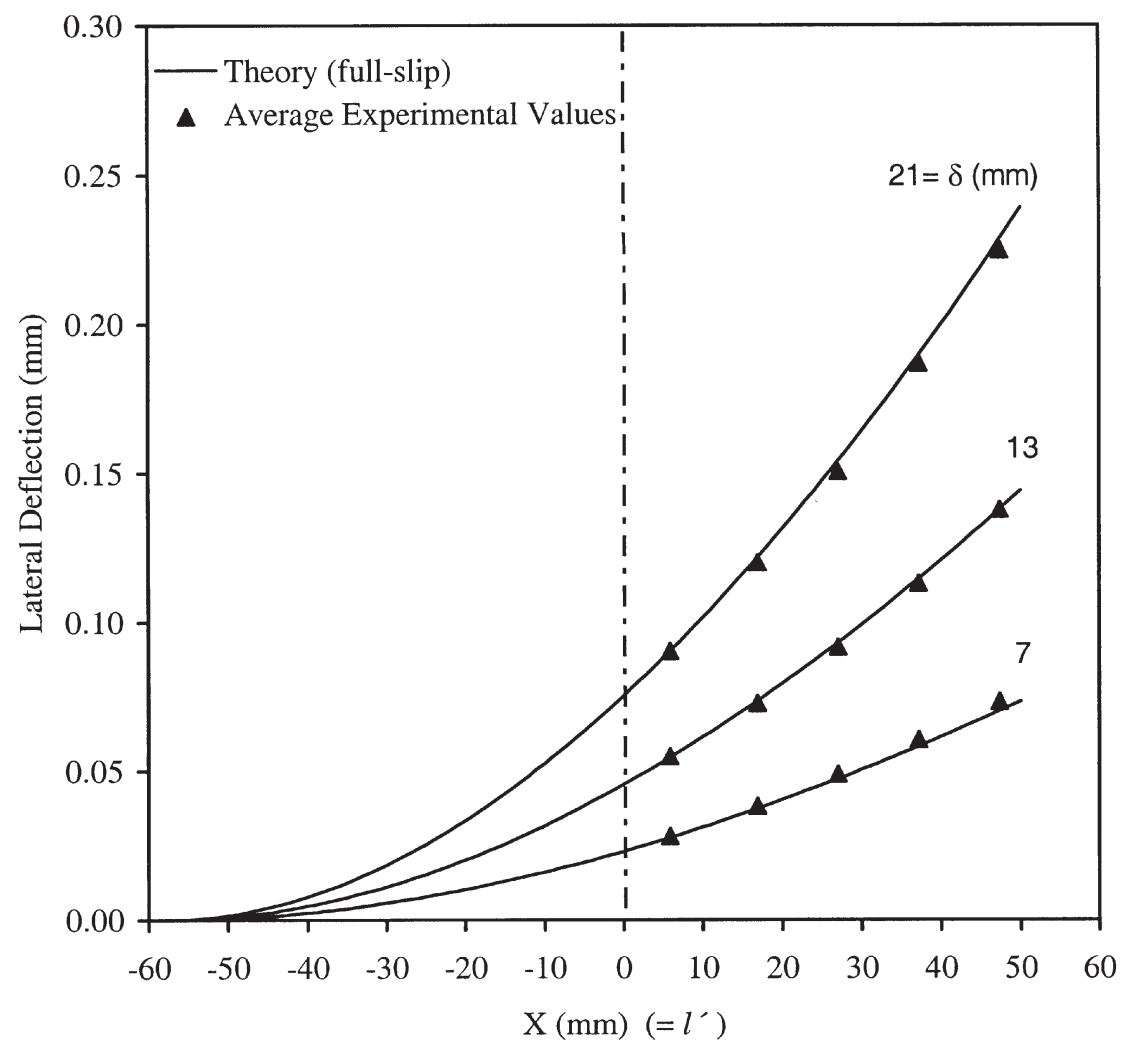

Fig. 6 Correlations between the experimental and theoretically obtained deflected shapes for the $39 \mathrm{~mm}$ strand at the propped socket under modes $(-\delta)$ and $(+\delta)$ of free bending, based on the full-slip $(E I)_{\text {eff }}$

conical housing), which is quite close to the value of $l^{\prime}=-58 \mathrm{~mm}$ associated with the corresponding theoretical full-slip $(E I)_{\text {eff }}$ case. With $\delta=21 \mathrm{~mm}$ and using the no-slip value of $(E I)_{\text {eff }}$, equation (6) gives $\rho=21 \mathrm{~m}$.

Based on Raoof 's experimental [11] and theoretical [3] studies, interlayer slippage in the immediate vicinity of fixed end starts around the neutral axis. The magnitude of at least the initial $(E I)_{\text {eff }}$ of the spiral strand, on the other hand, is a function primarily of the longitudinal wire stresses around the extreme fibre position, where the onset of variations (reductions) in the interlayer shear stiffness associated with reductions in the imposed radii of curvature is expected to lag considerably behind those around the neutral axis. It must be emphasized that, in view of the helical nature of the individual wires, in axially preloaded spiral strands the radial contact forces are a minimum at the interface between the outer and second (penultimate) layers and tend to increase substantially inwards, towards the core (King) wire where radial pressures become a maximum [14]. It, then, follows that interlayer slippage initially starts between the outer and penultimate layers and gradually moves inwards, towards the centre of the strand, with the proviso that it is certainly not impossible for the innermost layers to experience some form of interlayer slippage in the vicinity of the so-called neutral axis prior to the occurrence of interlayer slippage between the two outermost layers around the so-called extreme fibre position [6].

Extensive strain gauge readings in the immediate vicinity of the fixed ends to the present spiral strand of $39 \mathrm{~mm}$ diameter and another strand of $41 \mathrm{~mm}$ diameter, undergoing cyclic lateral deflections, support the above arguments $[\mathbf{5}, \mathbf{1 1}]$. Similar to the $39 \mathrm{~mm}$ strand, the $41 \mathrm{~mm}$ strand was also terminated with zinc-poured sockets to BS 463, except for an elongated jaw. Most importantly, the lay angles in the outer and penultimate layers of these two strands were -17.74 and $+16.45^{\circ}$, and +12.45 and $-11.96^{\circ}$ respectively, with the lower bound to the current manufacturing limits for the lay angle being $11^{\circ}$. The tests on these two strands (both of which were subjected to nominally identical testing conditions as in Fig. 2 with $x=2530 \mathrm{~mm}$ and $L=7.9 \mathrm{~m}[\mathbf{5}, \mathbf{1 1}])$ have clearly demonstrated that (for similar strand mean axial strains) the initiation of interlayer slippage associated with the strand of $41 \mathrm{~mm}$ diameter with smaller lay angles (cf. specimen of $39 \mathrm{~mm}$ diameter) occurs at larger radii of curvature; i.e. the lower the lay angle, the larger is the critical radius of curvature 
at the fixed end, $\rho$, at which interlayer slippage is initiated. In particular, with the strand of $41 \mathrm{~mm}$ diameter subjected to a mean axial load of $0.440 \mathrm{MN}$ (which with a full-slip effective axial stiffness $E_{\text {full slip }}=173.78 \mathrm{kN} / \mathrm{mm}^{2}$ corresponds to a mean axial strain $\varepsilon_{\mathrm{c}}=0.00239$ ), strain gauge readings in the immediate vicinity of the nominally fixed zinc socket (as presented in Fig. 10 of reference [11]) suggest that interlayer slippage around the so-called extreme fibre position initiates at a lateral deflection (at the lateral jack position) $\delta \approx 18 \mathrm{~mm}$ (Fig. 2). With $x=2530 \mathrm{~mm}, T=0.440 \mathrm{MN}$ and the theoretical fullslip bending stiffness for the $41 \mathrm{~mm}$ strand, $(E I)_{\mathrm{eff}}=1.809 \times 10^{10} \mathrm{~N} \mathrm{~mm}^{2} \quad$ [equation (6)] with $\delta=8 \mathrm{~mm}$ gives $\rho=25.9 \mathrm{~m}$; i.e. for $\varepsilon_{\mathrm{c}}=0.00239$ (for the strand of $41 \mathrm{~mm}$ diameter), plane-section bending may be assumed provided that $\rho>26 \mathrm{~m}$ (say) or $\rho / d>630$, where $d$ is the spiral strand's outer diameter.

Guided by the good correlations found between the theoretical predictions and the full range of test data, as presented in Fig. 6, and bearing in mind the above comments, it is concluded that for axially preloaded spiral strands with $\varepsilon_{\mathrm{c}}>0.0025$ (say) and outside diameters $d \leqslant 40 \mathrm{~mm}$, firstly for $\rho / d>630$, the use of the plane-section bending assumption for predicting the $(E I)_{\mathrm{eff}}$ and, hence, estimating the deflected shape of the spiral strand in the immediate vicinity of the assumed fixed end is reasonable and, secondly, the ideal point of end fixity, at least for this particular socket-strand system, is located inside the conical housing, at a distance from the face of the socket, $l^{\prime}$, of about $l^{\prime}=-1.5$ diameters.

In view of the extremely short length of the zone of influence of bending stiffness along the deflected shape of the spiral strand [which is located in the immediate vicinity of the end termination(s)], coupled with the fact that the position in practice of the point of end fixity is well inside the socket, it is not surprising that (bearing in mind the other problematic issues, as mentioned earlier in the introduction) developing a reliable design method against spiral strand restrained bending fatigue has (over the years) proved to be a very challenging task. It is encouraging, however, that (irrespective of the effective fixed end's exact location in practice) the present findings provide substantial support (within certain limiting conditions which for spiral strands with $d \leqslant 40 \mathrm{~mm}$ have now been reasonably identified) for the traditional (although previously not properly verified) approach for the determination of the minimum (critical) radius of curvature at the theoretically assumed (ideal) point of end fixity, $\rho$, with this parameter subsequently often used as an input into various available models for design against spiral strand restrained bending fatigue. It is now suggested that, in such cases, the critical portion of the spiral strand's deflected shape is merely shifted (without any change in form) along the strand, with the actual location of the effective fixed end being well inside the zinc socket at a distance from the socket face (i.e. the strand-zinc interface) which depends on the specific nature of the spiral strand-socket production procedure adopted in practice (including the quality of workmanship).

\section{CONCLUSIONS}

Using data from certain large-scale and carefully conducted cyclic free-bending experiments on an axially preloaded spiral strand of $39 \mathrm{~mm}$ diameter, in conjunction with a theoretical model, an insight is given into certain characteristics of the laterally deflected shapes of spiral strands in the immediate vicinity of zinc-socketed end terminations. Based on such a semi-empirical approach and guided by the previous experimental observations and theoretical work by the first author, it is concluded that, for spiral strands with mean axial strains $\varepsilon_{\mathrm{c}}>0.0025$ and outside diameters $d \leqslant 40 \mathrm{~mm}$, firstly, planesection bending may be reasonably assumed in the course of calculating the effective bending stiffness $(E I)_{\mathrm{eff}}$, with the proviso that the ratios of the estimated minimum radii of curvature at the fixed end(s) to the spiral strands, $\rho$, to the strand's outer diameter $d$ must be greater than 630 (i.e. $\rho / d>630$ ) and, secondly, for zinc-socketed terminations to such strands undergoing cyclic bending, the effective point of end fixity is located not at the face of the zincsocketed termination (i.e. the position where the spiral strand enters the zinc-filled conical housing), but well within the socket itself; for a strand of $39 \mathrm{~mm}$ diameter, the effective position of fixity was found to be located inside the conical housing, about 1.5 diameters away from the face of the socket.

The inevitable uncertainties as regards the exact location of the effective fixed end in practice (which very much depends on the specific nature of the strand-socket manufacturing procedure adopted) are, however, suggested not to have a practically significant adverse bearing on the accuracy of the traditional approach(es) for estimating the minimum (critical) radii of curvature, $\rho$, at the fixed end(s) with such values of $\rho$ often used as an input into various available models for design against spiral strand restrained bending fatigue.

\section{REFERENCES}

1 Raoof, M. Design of steel cables against free-bending fatigue at terminations. Struct. Engr, 1993, 71(10), 171178. 
2 Raoof, M., and Hobbs, R. E. The bending of spiral strand and armoured cables close to terminations. Trans. ASME, J. Energy Resources Technol., 1984, 106(3), 349355.

3 Raoof, M. Free-bending of spiral strands. J. Engng Mechanics Div., Am. Soc. Civ. Engrs, 1990, 116(3), 512530.

4 Raoof, M. Free-bending fatigue life estimation for cables at points of fixity. J. Engng Mechanics Div., Am. Soc. Civ. Engrs, 1992, 118(9), 1747-1764.

5 Raoof, M. Free bending fatigue of axially preloaded spiral strands. J. Strain Analysis, 1992, 27(3), 127-136.

6 Raoof, M., and Huang, Y. P. Free bending characteristics of axially preloaded spiral strands. Proc. Instn Civ. Engrs, Structs Bldgs, November 1992, 94, 469-484.

7 Poffenberger, J. C., and Swart, R. L. Differential displacement and dynamic conductor strains. IEEE Trans., Power Apparatus Systems, 1965, PAS-84, 281289.

8 Wyatt, T. A. Secondary stresses in parallel wire suspension cables. J. Struct. Div., Am. Soc. Civ. Engrs, 1960, 86(ST7), 37-60.

9 Raoof, M., and Huang, Y. P. Simplified methods for analysing steel strands. Struct. Engr, 1992, 70(22), 390397.

10 BS 463: Part I: 1958 Sockets for Wire Ropes, 1958 (British Standards Institution, London).

11 Raoof, M. Free bending tests on large spiral strands. Proc. Instn Civ. Engrs, Part II, December 1989, 87, 605626.

12 Irvine, M. Local bending stresses in cables. Int. J. Offshore Polar Engng, 1993, 3(3), 172-175.

13 Hobbs, R. E., and Smith, B. W. Fatigue performance of socketed terminations to structural strands. Proc. Instn Civ. Engrs, Part II, March 1983, 75, 35-48.

14 Raoof, M., and Hobbs, R. E. Analysis of multi-layered structural strands. J. Engng Mechanics Div., Am. Soc. Civ. Engrs, July 1988, 114(7), 1166-1182.

\section{APPENDIX}

\section{Notation}

$d \quad$ outer diameter of spiral strand

$(E I)_{\text {eff }}$ effective bending stiffness of the spiral strand

$l, y \quad$ system of coordinates with the $l$ axis coinciding with the line of action of the tension $T$ in the free field (i.e. away from the termination and not influenced by the end effects)

$l^{\prime}, y^{\prime} \quad$ system of coordinates at an angle $\psi_{0}$ with respect to the $l, y$ system of coordinates and with its origin at the point of intersection between the spiral strand's centre-line and the face of the socket

$L \quad$ length of the test specimen

$M \quad$ bending moment

$T \quad$ mean axial tension on the spiral strand

$x \quad$ distance between the position of the transverse point load and the ideal fixed end to the spiral strand

$X \quad$ coordinate along the spiral strand's centreline with the origin at the face of the socket

$\alpha \quad$ lay angle of the helix defining the centre-line of an individual wire

$\delta \quad$ lateral deflection at the position of the transverse point load

$\varepsilon_{\mathrm{c}} \quad$ mean axial strain on the spiral strand

$\rho \quad$ radius of curvature at the fixed end, where $l=0$

$\psi_{0} \quad$ rotation of the tangent at the termination relevant to the direction of the tension in the spiral strand and the angle of rotation between the two systems of coordinates $l, y$ and $l^{\prime}, y^{\prime}$ 